\title{
Collective Properties of X-ray Binary Populations of Galaxies II. Pre-Low-mass X-ray Binary Properties, Formation Rates, and Constraints
}

\author{
H. Bhadkamkar ${ }^{1,2}$ and P. Ghosh ${ }^{1}$
}

Received __; accepted _

\footnotetext{
${ }^{1}$ Department of Astronomy \& Astrophysics, Tata Institute of Fundamental Research, Mumbai 400 005, India

${ }^{2}$ Astronomy \& Astrophysics, Raman Research Institute, Bengaluru, 560080
} 


\begin{abstract}
We continue exploring our understanding of the collective properties of X-ray binaries in the stellar fields (i.e., outside globular clusters) of normal galaxies, introduced in Paper I of this series, where we considered high-mass X-ray binaries (HMXBs). In this paper (Paper II of the series) and the companion paper (Paper III of the series), we consider low-mass X-ray binaries (LMXBs), whose evolutionary scenario is very different from that of HMXBs. In this paper, we consider the evolution of primordial binaries upto the stage where the neutron star just formed in the supernova explosion of the primary is in a binary with its low-mass unevolved companion, and this binary has circularized tidally, producing what we call a pre-low-mass X-ray binary (pre-LMXB). We study the constraints on the formation of such pre-LMXBs in detail (since these are lowprobability events), and calculate their collective properties and formation rate. To this end, we first consider the changes in the binary parameters in the various steps involved, viz., the common-envelope (CE) phase, the supernova, and the tidal evolution. This naturally leads to a clarification of the constraints. We then describe our calculation of the evolution of the distributions of primordial binary parameters into those of the pre-LMXB parameters, following the standard evolutionary scenario for individual bianries. We display the latter as both bivariate and monovariate distributions, discuss their essential properties, and indicate the influence of some essential factors on these. Finally, we calculate the formation rate of pre-LMXBs. The results of this paper are used in the next one (Paper III) to compute the expected X-ray luminosity function (XLF) of LMXBs, which is compared with observation.
\end{abstract}

Subject headings: binaries: close - stars: evolution - stars: neutron - stars: low-mass 
- supernovae: general - X-rays: binaries - X-rays: galaxies 


\section{Introduction}

In this series of papers, we are attempting a pioneering exploration of the essential theoretical underpinnings of the observed distributions of the collective properties of accretion powered X-ray binaries, in particular their X-ray luminosity functions (XLFs). Paper I (Bhadkamkar \& Ghosh 2012) of the series dealt with high-mass (or massive) X-ray binaries (HMXBs). In the next two papers of the series, we are dealing with low-mass X-ray binaries (LMXBs). It is convenient to divide this LMXB study into two parts, viz., (a) that which deals with the evolution of a primordial binary upto the formation of a neutron star in a supernova (SN) explosion, which produces a binary consisting of this neutron star and a relatively low-mass companion, which we call a pre-low-mass X-ray binary (pre-LMXB), and (b) that which deals with the evolution of this pre-LMXB into an X-ray active LMXB, and the subsequent evolution of this LMXB through its accretion phase. Accordingly, we handle the first part in this Paper, which is Paper II in this series. The second part is handled in a companion paper, which is Paper III in this series.

We briefly recount a few essential features of studies such this. First, such studies have become meaningful only in recent years, when it became possible to construct robust and dependable distributions of the essential collective properties of X-ray binaries, e.g., their XLFs, after the accumulation of four decades of observational material (Grimm et al. 2002, 2003; Kim \& Fabbiano 2004; Gilfanov 2004; Gilfanov et al. 2004a.,b; Liu et al. 2007;

Kim \& Fabbiano 2010). Second, in our study contained in this series of papers, we focus on X-ray binaries which are outside globular clusters, i.e., in the stellar field of the galaxy under study, which implies that the evolution of a given X-ray binary can be treated in isolation, without any significant perturbation from other stars or X-ray binaries. This is why the standard scenarios of individual X-ray binary evolution (van den Heuvel 1983, 1991, 1992, 2001) can be applied to the problem. Third, the multi-step evolutionary sequence from a 
primordial binary to an X-ray binary may involve both (a) steps in which only the initial and final states matter for our purposes, and (b) steps in which not only the initial and final states but also the entire intermediate process of evolution have to be considered for our purposes. Naturally, the second situation leads to more involved calculations. We showed in Paper I that the entire HMXB evolutionary process is described by the first situation. We shall show in this paper (Paper II) that the evolution from primordial X-ray binaries to pre-LMXBs is also almost described by the first situation, with one exception which is easily handled. By contrast, the subsequent LMXB evolution is almost entirely described by the second situation, and so has to be handled in a very different manner. This is a major reason why we chose to give it in a separate paper, viz., Paper III. Fourth, as with the HMXB work of Paper I, an effort of this type should be regarded only as a proof-of-principle demonstration that the observed X-ray-binary collective properties can be (at least) qualitatively accounted for by evolving well-known, plausible collective properties of primordial binaries through the well-known and well-accepted scenarios for the evolution of an individual primordial binary into an X-ray binary.

The rest of this paper is arranged as follows. In Sec2, we briefly recount the formation and evolution scenarios of pre-LMXBs and LMXBs. In Sec.3, we describe the changes in the binary parameters in various steps of primordial-binary evolution leading upto pre-LMXB formation, taking in turn the common-envelope (CE) phase, the supernova, and the tidal evolution. In Sec,4, we describe the constraints on the pre-LMXB parameters. In Sec.5, we describe our calculation of the distribution of pre-LMXB parameters. We start from a summary of canonical primordial parameter distribution, and we describe how we transform this distribution to obtain that for pre-LMXBs. We show the latter as both bivariate and monovariate distributions, discuss their properties, and show the influence of some essential factors on these. In Sec 6 , we present our calculation of the formation rate of pre-LMXBs. We discuss our results in Sec:7. 


\section{LMXB Formation and Evolution Scenarios}

We briefly recount here the standard formation scenario for LMXBs. Their progenitors are primordial binaries of two main sequence stars, which are much more disparate in mass than those in HMXB progeniors. This extreme mass ratio changes the course of LMXB formation and evolution completely from that of HMXBs. The more massive star, i.e., the primary, completes its main sequence life faster, on timescales $\sim 10^{6}-10^{7} \mathrm{yr}$. On the giant branch, it fills its Roche lobe and starts transferring mass to the secondary, which, in such a progenitor, is so much less massive than the primary, and hence has so much longer a thermal timescale than the primary, that it is unable to accept the transferred matter, which then forms an envelope around the two stars - the common envelope (CE). The primary keeps losing mass to form this CE until it is completely stripped of its H-envelope. The CE, which engulfs the resulting binary of the He-core of the primary and the secondary, exerts a strong frictional drag on this binary, so that the two stars spiral in towards each other. The orbital energy released due to this spiral-in is deposited into the CE, heats it and attempts to expel it. Systems which were very close to begin with now go into a merger and so do not survive this phase, but those which were sufficiently wide do have enough energy to expel the CE altogether, and so survive the CE phase, emerging as a very compact binary consisting of the He-core of the primary and the secondary, the latter remaining practically unchanged during this whole process.

The post-CE system is typically detached and the He-core evolves as if it were a single He-star, exploding eventually as a supernova (SN). A neutron star of typical mass $1.4 M_{\odot}$ is left after the SN explosion of the He-core, the rest of the mass being lost from the system. Due to the large mass loss from the system during the previous CE phase, the mass loss in this SN event is not always destructively large, and some of these systems do survive, but often with rather large eccentricity. The rest of the systems are disrupted. Natal kicks are 
given to the neutron star when the SN explosion is asymmetric, and these further influence the survival probability of these post-CE binaries. The resultant binary, consisting of the neutron star and its low-mass companion, now evolves tidally on timescales $\sim 10^{5}-10^{6} \mathrm{yr}$, circularizing the binary. The whole sequence of events outlined above occurs over $\sim 10^{7}$ yr, which is very short compared to the nuclear timescale of the secondary, which for a typical secondary mass of $1 M_{\odot}$ is $>10^{9}$ years. Therefore, the secondary undergoes little nuclear evolution during the above time, and can still be considered almost a Zero Age Main Sequence (ZAMS) star. We call this binary system of a neutron star and low-mass unevolved companion a Pre-Low-Mass X-ray Binary (pre-LMXB).

LMXB formation and evolution can thus be thought of as a two-step process. The first step is the rapid one outlined above, occurring over timescales of $\sim 10^{7} \mathrm{yr}$. The second step is a very slow one, occurring over timescales $\sim 10^{9}$ years, during which (a) the above pre-LMXB evolves through its detached binary phase to the onset of Roche-lobe overflow and mass transfer, at which point the system turns on as a LMXB, and (b) this LMXB lives out its X-ray emission phase and turns off, after which system evolves into a binary consisting of a recycled neutron star with a white dwarf companion. We treat the first step in detail in the rest of this paper (Paper II), deferring a detailed treatment of the second step to the next paper of this series (Paper III), and giving only a very brief outline of that step in the next paragraph.

The pre-LMXB evolves through the angular momentum loss which causes its orbit to shrink, the two major mechanisms for such loss being gravitational radiation and magnetic braking. The first is always in operation, though not always dominant. Magnetic braking is operational when the companion has a sufficiently large and sustained magnetic field, and a magnetically coupled stellar wind carries off significant angular momentum. This is thought to require both a sizable radiative core (to anchor the magnetic field) and a 
sizable convective envelope (to run the dynamo that produces the magnetic field) in the companion, a point to which we return later. Both mechanisms have strengths which increase rapidly with decreasing orbital radii, and so are important only at small radii. Accordingly, these mechanisms can bring the system into Roche-lobe contact within the main-sequence lifetime of the low-mass companion only if the initial orbital radius of the pre-LMXB is sufficiently small. For such systems, Roche-lobe overflow is established while the companion is still on the main-sequence, and the subsequent mass transfer is driven by angular-momentum loss. For wider systems, however, Roche-lobe contact becomes possible only after the companion finishes its main-sequence lifetime, gets on the giant branch and expands to fill its Roche lobe. Subsequent mass transfer is driven dominantly by the nuclear evolution and expansion of the companion. The crossover point between the above two possible evolutionary paths occurs at a critical initial orbital period called the bifurcation period, whose value is $\sim 14-18$ hours (Pylyser \& Savonije 1988, 1989). In either case, the matter transferred to the neutron star's Roche-lobe forms an accretion disk and eventually reaches the surface of the neutron star, the energy released in the process being emitted in X-rays, making the system a bright LMXB. This LMXB phase ends when accretion stops and the companion becomes a degenerate white dwarf, the final product being a recycled neutron star in either (a) a close orbit with either a low-mass He white dwarf or a somewhat heavier CO white dwarf, or (b) a wide orbit with a low-mass He white dwarf. The former class comes from the angular-momentum-loss driven evolution of systems below the bifurcation period, while the latter class comes from the companion-nuclear-evolution driven evolution of systems above the bifurcation period.

We note here that other scenarios for the formation of LMXBs have been suggested, e.g., accretion induced collapse of a white dwarf. (See,e.g., Kalogera \& Webbink (1998); Kalogera (1998) and references therein). However, most of the scenarios relevant for the LMXB systems in the field of the galaxy invoke CE scenario and hence fall in the general 
class whose evolutionary sequence is similar to what we described above. Further, since we are interested here in the LMXB populations outside globular clusters, we do not consider those scenarios which invoke stellar encounters as the dominant mechanism for the formation of these systems.

\section{Evolutionary Changes in Binary Parameters}

\subsection{The common envelope (CE) phase}

The conditions required for the formation of a CE have been studied in great detail in the literature (for detailed reviews, see, e.g., Taam \& Sandquist (2000); Webbink (2008)). The essential ingredients for the viable formation of a CE are (a) an extreme mass ratio and (b) an evolved primary. Depending upon the evolutionary stage of the primary, three regimes of the primary's Roche-lobe overflow, namely Cases A, B and C, have been defined and are widely used in the literature. The cases of interest to us here usually belong to Cases B and C, involving an evolved primary. (Case A occurs when the donor is on main sequence, which can happen in very close primordial binaries, but this case is not of interest here, since it would lead to a merger during spiral-in, as explained earlier). The other condition, i.e., that of extreme mass ratio, is usually taken as $q<0.3$, because in this regime the thermal timescales of the two stars are different by an order of magnitude or more. This regime of $q$-values is completely disjoint with that relevant for the HMXB progenitors (see Paper I), as expected. The mass of the secondary remains unchanged during the entire evolution from the primordial binary to Roch-lobe overflow, as explained earlier. The upper limit to this mass is usually taken as $1-2 M_{\odot}$, although some authors have suggested

somewhat higher limits (Podsiadlowski et al. 2002; Pfahl et al. 2003), in which case there is expected to be considerable mass loss in the initial phases of LMXB operation. For typical primary masses in primordial binaries, which are in the range $9-30 M_{\odot}$ for LMXBs, the 
mass ratio $q$ thus always satisfies the above criterion of extremeness.

Quantitative descriptions of the binary-parameter changes in the CE phase can be given from the general picture of CE evolution given above (Webbink 1984). The mass of the common envelope is usually taken as the mass of the primary envelope, as only the He-core of the primary is left at the end of the CE phase. The energy deposited in the CE is equal to the difference in the binding energy of the pre-CE and post-CE system, multiplied by an efficiency factor $\alpha$. This energy must be equal to the core-envelope binding energy for the primary, if the enevelope is to be expelled finally. Thus, if $E_{b 1}$ and $E_{b 2}$ are the initial and final orbital binding energies and $E_{c-e}$ is the energy of core-envelope binding, the energy equation describing CE process is $\alpha\left(E_{b 1}-E_{b 2}\right)=E_{c-e}$. Now, the binding energy $E_{b}$ can be written as $G M_{1} M_{2} / 2 a$ where the masses and the distance are taken appropriately. Calculation of $E_{c-e}$ requires a knowledge of the detailed structure of the star, and the result can be parametrized as $E_{c-e}=G M_{p, c} M_{p, e} / \lambda R$ where $M_{p, c}$ and $M_{p, e}$ are the masses of the core and the envelope of the primary respectively and $R$ is the radius of the star. Details of stellar structure are contained in the parameter $\lambda$.

Since the radius of the star equals that of its Roche lobe at the time of CE formation, the relation between the initial and final orbital separations can be written as

$$
\frac{a_{C E}}{a_{0}}=\frac{M_{p, c} M_{s}}{M_{p}} \frac{1}{M_{s}+2 M_{p, e}\left(\alpha \lambda r_{L}\right)^{-1}} .
$$

The relation between $M_{p, c}$ and $M_{p}$ is often taken as a power-law approximation to the results of detailed stellar-structure calculations, given by

$$
M_{p, c}=M_{0} M_{p}^{1 / \xi}
$$

where $M_{p, e}=M_{p}-M_{p, c}$. Here, $r_{L}$ is the effective Roche lobe radius of the primary. It depends only upon the mass ratio $q \equiv M_{1} / M_{2}$, and analytical approximations to the numerical results are available in the literature. We adopt here the widely-used Eggleton 
approximation (Eggleton 1983), which is

$$
r_{L}=\frac{0.49 q^{2 / 3}}{0.6 q^{2 / 3}+\ln \left(1+q^{1 / 3}\right)} .
$$

From the above energy equation, it is clear that the orbital shrinkage during the CE phase depends upon the product $\alpha \lambda$ of the two essential parameters of the problem. Consequently, a modeling of the CE process alone cannot give independent handles on these two parameters, but can only constrain their product. However, many authors have suggested constraints on the values of $\alpha$ and $\lambda$, which are based on observations of CVs and LMXBs, and also from the results of stellar-evolution computations. From these works it is seen that, although values of $\lambda$ can be estimated with reasonable confidence for a large range of stellar parameters, $\alpha$ is still rather uncertain, with expected values $\sim 0.5-1$ (Dewi \& Tauris 2000; Willems \& Kolb 2004; De Marco et al. 2011; Ivanova 2011). Since independent constraints are not possible from CE modeling alone, as in this work, we here work only with the product $\alpha \lambda$, calling it the $C E$ parameter.

The transformation connecting the parameters of the primordial binary to those of the post-CE binary are given by Eqs. 1 and 2 along with the definition $M_{s}=q M_{p}$. The inverse transformation can be derived from these, and the Jacobian for that transformation is given by

$$
J_{C E}=\frac{\xi}{M_{p, c}} \frac{a_{0}}{a_{C E}}
$$

\subsection{Supernova}

The detached post-CE system evolves at first without affecting the binary parameters. Then the He-core of the primary finishes its evolution like a single He-star and explodes as a SN, which changes the binary characteristics suddenly. Throughout this paper, as well as Paper III, we assume that the neutron star formed in the SN explosion has a mass 
of $1.4 M_{\odot}$ (as we did in Paper I) and rest of the mass of the He-core is lost from the system. If this mass loss is sufficiently large (which it can be depending on the details of the primordial binary, despite the heavy mass loss from the primary during the previous CE phase), the binary is disrupted. If the SN explosion is symmetric, a mass loss of more than half of the total mass of the system will unbind the binary. For smaller mass losses, the parameters of the post-SN binary are related in a simple way to those of the pre-SN binary. However, natal kicks given to the neutron star due to asymmetric SN explosions alter this simple picture considerably. A quantitative general treatment of the binary-parameter changes including all of the above points has been given in Sec.3.2 of Paper I, details of the computation (particularly those of the averaging process over the distribution of natal kicks) being given in Appendix A of that paper.

We continue here with the notation introduced in the above places in Paper I. In particular, in averaging over the 3D isotropic Maxwellian distribution $\propto v^{2} \exp \left(-v^{2} / 2 \sigma^{2}\right)$ of the natal kick velocities $v$, we introduced an upper limit $v_{u p}$ where we truncated the processes of averaging over this kick-velocity distribution (see Eq.(A2) of Paper I): this limit corresponds to the point at which the post-SN system becomes just unbound (see Eq.(A1) of Paper I), so that extending the integration above this limit would incorrectly include the unbound systems also, which in reality must be excluded. Further, for algebraic convenience, we expressed this upper limit in the form $v_{u p} \equiv(\sqrt{2} \sigma) f$, and worked with the parameter $f$. As shown in Appendix A of Paper I, the distribution-averaged kick-velocity square $v_{k}^{2}$ could then be expressed in the simple form $v_{k}^{2}=\sigma^{2} h(f)$, where $h(f)$ was a function of $f$ alone. As detailed there and in Fig.13 of Paper I, two regimes of behavior were clearly shown by $h(f)$ : for $f<f_{c}, h(f) \propto f^{2}$, and for $f>f_{c}, h(f) \approx$ constant, with a critical value $f_{c} \approx \sqrt{2.5}$. The transition region between the two regimes is very narrow. This clear separation of regimes made the calculation of $v_{k}^{2}$ easy. 
Here in the case of pre-LMXBs, $v_{u p}$ has a relatively large range $\sim 50-400 \mathrm{~km} / \mathrm{s}$. But the values $\sigma=26.5 \mathrm{~km} / \mathrm{s}$ and $\sigma=265 \mathrm{~km} / \mathrm{s}$, corresponding to ECSN and ICCSN respectively, still give values of $f$ which are on opposite sides of $f_{c}$. Due to the extremely narrow transition region in $f$, these two cases can, therefore, still be treated in a way similar to that used in the treatment used for HMXBs in Paper I. Detailed calculations show that $\sigma=26.5 \mathrm{~km} / \mathrm{s}$ gives results extremely close to no-kick case. Therefore, for our LMXB work here we need only calculate the large-kick ICCSN case, i.e., $\sigma=265 \mathrm{~km} / \mathrm{s}$, explicitly in addition to the no-kick case, since the latter serves as an excellent description of the small-kick ECSN case. The Jacobian $J_{S N}$ calculated in Appendix A.2 of Paper I can be used directly in this work for appropriate values of the relevant parameters.

\subsection{Tidal evolution}

The post-SN binary of the neutron star and its low-mass companion is typically very eccentric, as we show in sec. 5.2. Such a system evolves due to tidal interaction on timescales of $\sim 1$ Myr or less. Tidal interaction has three effects on the binary: (a) circularization, (b) synchronisation and (c) change in the semimajor axis. Tidal evolution of binaries has been much studied in the literature, (Zahn 1977; Hut 1981), particularly in the context of LMXBs (Kalogera 1996). We treat tidal effects in pre-LMXBs in this work with the aid of the prescription given by Hut, which can be used in case of large eccentricities. Due to the extreme compactness of the neutron star, it does not take part in the tidal evolution. Further, since the orientation of the orbit is inconsequential for our purposes here, we consider only the changes in the eccentricity and semimajor axis of the orbit and those in the rotation of the companion. Finally, we note that we are interested here not in the detailed evolution of these parameters, but rather in the relations between their initial and final values. Such relations can be obtained by noting that the tidal evolution 
preserves the total angular momentum of the system. We note here that Kalogera (1996) also treated tidal evolution similarly. However, we also try here to incorporate the effect of the companion's spin through the exchange of angular momenta between the orbital motion and the spin of the companion. Thus the effect of the tidal evolution can be schematically written as $J_{\text {orb }}+J_{s, \text { spin }}=$ constant. Using standard expressions for orbital and spin angular momenta, this relation can be re-expressed as

$$
F\left[\alpha^{-1 / 3}\left(1-e^{2}\right)^{1 / 2}-1\right]=n_{f}^{4 / 3}(1-\alpha k)
$$

Here $\alpha \equiv n_{i} / n_{f}, k \equiv \Omega_{i} / n_{i}$, and $n_{i}$ and $n_{f}$ are the average angular velocities in the initial and final states, given by $n=\sqrt{G\left(M_{N S}+M_{s}\right) / a^{3}}$, with the masses and semimajor axes appropriate for the initial and final states respectively. Since the masses do not change during tidal evolution, $n_{i}$ and $n_{f}$ differ only through the values of semimajor axes, so that their relation gives that between the initial and final semimajor axes, provided that other quantities are specified. $\Omega_{i}$ is the initial spin angular velocity of the companion. It is assumed that $\Omega_{f}=n_{f}$ and $e_{f}=0$, i.e., the binary is circular and the companion is rotating synchronously at the end of the tidal evolution. $\Omega_{i}$ and therefore $k$ can be treated as a free parameter in our calculations, with an allowed range $0<k<k_{\max }$, where $k_{\max }$ represents a maximally spinning companion just after the SN. Numerical calculations show that value of $k$ does not change $\alpha$ by a large amount. The most likely value of $k$ is $k_{C E}=\sqrt{1-e^{2}} /(1-e)^{2}$. Since the CE process applies a large frictional drag on the binary, we can safely assume that the companion's spin period equals the orbital period at the end of the CE phase. Thus $n_{C E}=\Omega_{C E}=\Omega_{i}$ where $\Omega_{C E}$ and $n_{C E}$ are the spin and the orbital angular velocities at the end of the CE phase respectively. $F$ in Eq.(55) is a function of the two masses and hence is constant for a given system. It is given by

$$
F=\frac{G^{2 / 3} M_{N S} M_{s}}{\left(M_{N S}+M_{s}\right)^{1 / 3} I_{s}},
$$

where $I_{s}$ is the moment of inertia of the secondary. It can be written in terms of solar units 
as $I_{s}=i_{s} M_{\odot} R_{\odot}^{2}$. The scaling of $i_{s}$ with the stellar mass is given by Rucinski (1988). In this work, we use an analytical fit to their data which is given by $i_{s}=0.0832 M_{s}^{2.27}$.

Solving Eq.(5) for $\alpha$ gives the relation between orbital separations before and after the tidal evolution. The companion mass $M_{s}$ is of course unchanged during the process, and the initial eccentricity is taken as the third dummy parameter for calculational ease. Thus the transformation is essentially only in one parameter, i.e., the orbital separation, described by $\alpha$. The Jacobian for the inverse transformation is then given by

$$
J_{\text {tid }}=\sqrt{\frac{a_{p s n}}{a\left(1-e^{2}\right)}}\left[1-\frac{3}{f} n_{f}^{4 / 3}\right] .
$$

\section{Constraints on Pre-LMXB Parameters}

Formations of LMXBs are very low-probability events. Various system parameters need to have values in very narrow ranges for the binary to survive through the various phases of the evolutionary process. Constraints on the allowed ranges of these binary parameters at different stages of evolution can be translated to those on primordial binary parameters, using the transformations described in Sec 3 . Such constraints were first discussed by Kalogera \& Webbink (1998). Let us first study the constraints which are relevant at pre-LMXB stage, identify the allowed zones, and calculate the probability of formation. These constraints can be expressed as follows:

1. The primary must fill its Roche lobe. This ensures the formation of a CE, which is an essential step towards pre-LMXB formation. In this work, we adopt a very conservative limit for this by setting $a_{0} r_{L}(1 / q)=R_{B A G B}$, where $R_{B A G B}$ is the radius of the primary at the base of the asymptotic giant branch. Analytical fits given by Hurley et al. (2000) are used to calculate $R_{B A G B}$. This gives an upper limit on $a_{0}$ as a function of the metallicity of the primary for given primary and secondary masses. 
Fig. 1(a) shows this upper limit as a function of the mass of the secondary, for $M_{p}=12 M_{\odot}$. A representative lower limit for $\alpha \lambda=1$ is also shown. It can be easily seen that higher metallicity allows higher phase-space area, thus producing a larger number of systems, although this condition is not very restrictive.
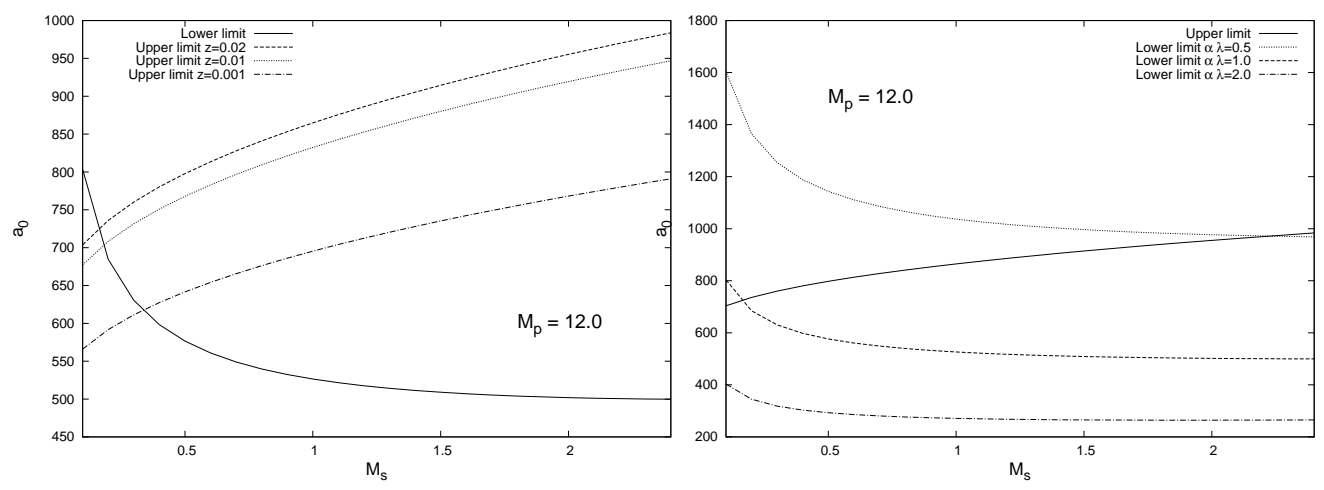

Fig. 1.- Constraints on $a_{0}$ as a function of $M_{s}$. (a) left panel: upper limit for different $z$ values. (b) right panel: lower limit for different CE-parameter values. Both plots for $M_{p}=12 M_{\odot}$.

2. The post-CE system must be detached. This ensures uninterrupted evolution of the He-core of the primary, resulting in the formation of a neutron star. This is a twofold constraint, requiring that the radii of both the secondary and the He-core must be less than their respective Roche-lobe radii. Although this constrains the post-CE orbital separation from below, we can convert it to a constraint on $a_{0}$ using eqn. 11. This constraint depends, of course, upon the value of the CE parameter, as shown in Fig.1(b). Note that a representative upper limit, corresponding to $z=0.02$ (taken from the left panel (a) of this figure), is repeated in this panel for comparison, and similarly a representative lower limit, corresponding to $\alpha \lambda=1$ (taken from this panel), is repeated in the left panel (a) of this figure for the same purpose.

It is clear from the above that very inefficient $\mathrm{CE}$ processes prohibit most of the parameter space, since the lower limit is larger than the the upper limit over a large 
range of $M_{s}$. For values of the CE parameter above unity, however, a large range is allowed and the constraints are not very restrictive. We also note that changes in the $\mathrm{CE}$ parameter make larger changes in the allowed range of $a_{0}$, making it the more dominant parameter in determining the PDF.

3. The binary must survive the SN explosion. Disruption of the binary due to sudden mass loss is discussed above in Sec 3.2 , with reference to detailed calculations in Paper I. This requirement puts a lower limit on the allowed values of $M_{s}$, or equivalently on those of $q$, as functions of $M_{p}$. We set an absolute lower limit on $M_{s}$ as $0.1 M_{\odot}$. Thus the limit obtained from the condition of SN survival is applicable only above a certain value of $M_{p}$, below which the entire range is allowed. We set the upper limit on $M_{s}$ as $2.5 M_{\odot}$ matching with the constraints used by Grimm et al. (2002). Fig,2 depicts these constraints. It can be seen that, for neutron-star LMXBs, the progenitor primary must be less massive than $\approx 21 M_{\odot}$.

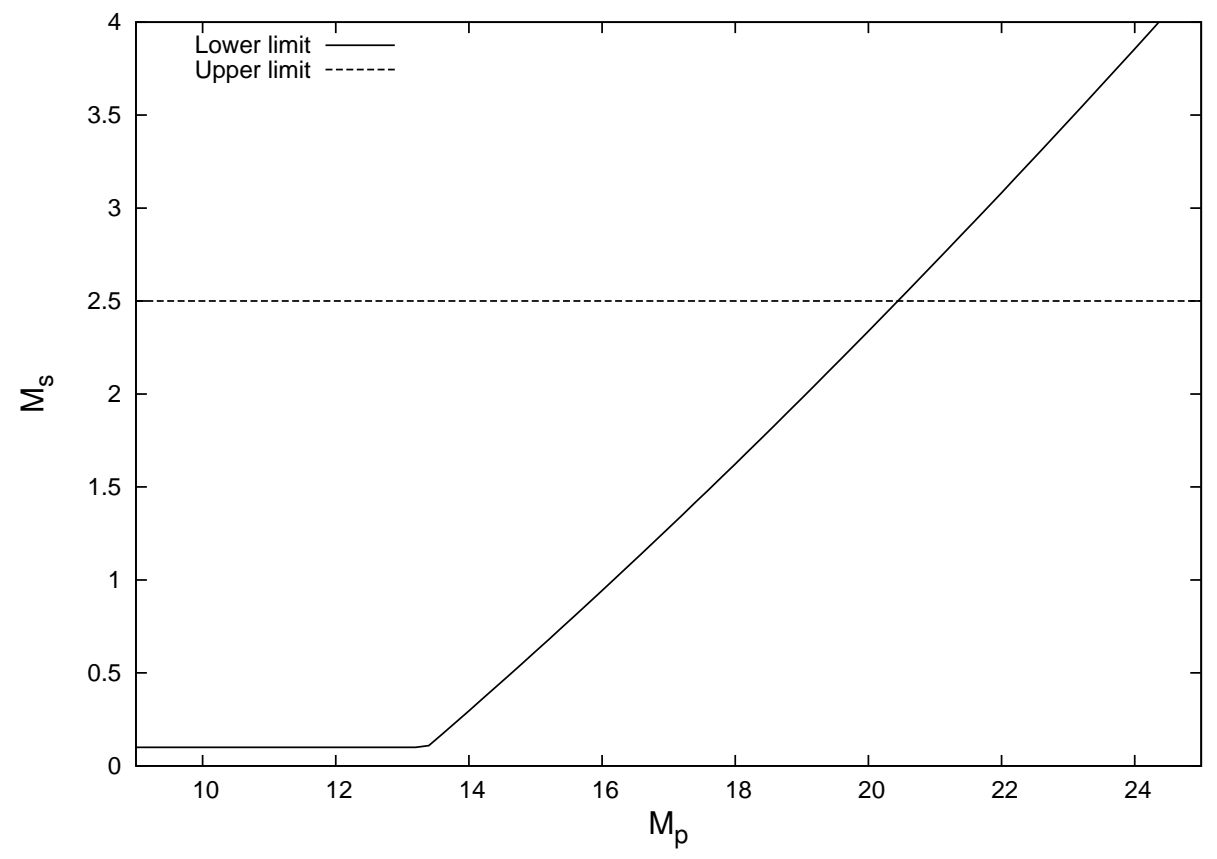

Fig. 2.- Constraints on the allowed range of $M_{s}$ as a function of $M_{p}$. 


\section{Pre-LMXB Parameter Distribution}

\subsection{Distribution of primordial binary parameters}

Primordial binary distributions for LMXB progenitors are characterized by three parameters, viz., the primary mass $M_{p}$, the secondary-to-primary mass ratio $q$, and the orbital separation $\left(a_{0}\right)$, as for the HMXB progenitors considered in Paper I. These three parameters essentially follow the same distribution as in case of HMXBs, i.e., an IMF describing the distribution of $M_{p}$, a power-law (flat or falling) distribution for $q$ and Öpik's law for $a_{0}$. But the allowed ranges for some of these parameters are drastically different from those that apply to HMXB progenitors. Especially, we note that the $q$-ranges for the two cases are completely disjoint, as expected from the thermal-timescale arguments. Also, the range of $a_{0}$ is more tightly constrained in the case of LMXBs due to stricter conditions of survival through various phases, as described in Sec 4 ,

The requirement of a viable formation of CE constrains $q$ from above as $q<0.3$, which is an absolute upper limit. This allows us to have binaries with companion masses such that the some of the final products may be more appropriately called Intermediate Mass X-ray Binaries (IMXBs), in addition to LMXBs. The upper limit for the companion mass is generally taken to be in the range $1-3 M_{\odot}$ if the binary is to be classified as an LMXB. We take this upper limit as $2.5 M_{\odot}$ following Grimm et al. (2002). This puts another upper limit on $q$ given by $2.5 / M_{p}$. We find that, for the entire range of $M_{p}$, this limit is always lower than the limit imposed by CE formation given above. However, the distribution of

$q$ is poorly constrained within this range. It has been suggested by Sana \& Evans (2010) that the uniform $q$-distribution observed for $q>0.2$ can be extended to the lower $q$-values. For LMXB/IMXB population studies, this uniform $q$-distribution has been indeed been used by Pfahl et al. (2003). By contrast, a $q^{-2.7}$-distribution, i.e., a rather steeply falling power-law, was used in the comprehensive LMXB work of Kalogera \& Webbink (1998). We 
in this work use the general form $\propto q^{\beta}$ and take $\beta=0$ and $\beta=-2.7$ to accomodate both the approaches mentioned above, as also to explore any other power-law, if necessary.

The distribution of the orbital separation is taken as Öpik's law, (Öpik 1924) as per current norm. We note however that the tight constraints in the LMXB-formation process described in section 4 severely limit the allowed range of $a_{0}$ which can eventually produce viable pre-LMXBs. It is not impossible that fluctuations within this small range may have been overlooked while deriving Öpik's law for a much wider range of separations. However, due to the lack of a close coverage of data over small ranges of separations, we find it most prudent to assume at this time that the wide-range log-uniform distribution also applies over the smaller range relevant for our purposes here. At a minimum, it certainly gives an indication of the average trend.

The distribution of the primary mass is given by the IMF (Salpeter 1955); Kroupa et al. 1993; Kroupa \& Weidner 2003). We in this work use the IMF given by Kroupa \& Weidner since it is applicable for a relatively wide range of masses and can be extended to the lowest mass ranges relevant for the secondary in these calculations.

The PDF of the primordial binaries can therefore be written as

$$
f_{\text {prim }}\left(M_{p}, q, a_{0}\right)=\frac{1}{N} \frac{\operatorname{IMF}\left(M_{p}\right) q^{\beta}}{a_{0}} .
$$

Here, $N$ is the normalization parameter defined such that when the above PDF is integrated over the relevant ranges of all the parameters, it yields unity.

\subsection{Transformation of PDF to pre-LMXB stage}

The Jacobian formalism described in Paper I can be used to transform the distribution of primordial binaries to the distribution of pre-LMXBs. Formation of pre-LMXBs proceeds through the three stages of parameter changes described in Sec.3. The binary at each stage 
is defined by three parameters, which can be connected to the parameters of the next stage. We start with the transformation from primordial binary to post-CE binary. The three parameters describing the primordial binary are $\left(M_{p}, q, a_{0}\right)$ and those describing the post-CE binary are $\left(M_{p, c}, M_{s}, a_{C E}\right)$. The transformation relations are given by Eqs. 1, 2 and the relation between $q$ and $M_{s}$ is of course $M_{s}=q M_{p}$. The Jacobian for the inverse transformations is given by Eq.(44).

The next stage of parameter change occurs at the SN explosion. The post-SN binary is described by $\left(M_{s}, a_{p s n}, e\right)$. We first note that $M_{s}$ is unchanged during this transformation. The inverse transformations for the other two parameters and the Jacobian for that are described in Appendix A of Paper I. As noted in Sec.3.2, we perform the calculations for ICCSN $\sigma=265 \mathrm{~km} / \mathrm{s}$ case and for the no-kick case, which also represents the ECSN case with $\sigma=26.5 \mathrm{~km} / \mathrm{s}$.

For the post-tidal binary, only two parameters, viz., $\left(M_{s}, a\right)$ are sufficient to describe the system. To describe the immediate post-SN system, however, we also need the orbital eccentricity e, i.e., a total of three parameters. We do the transformation of parameters and distributions between pre- and post-SN systems as described, and then integrate over the eccentricity in order to arrive at the post-tidal binary parameters. The distribution of the immediate post-SN eccentricity is shown in Fig. The small kicks characteristic of ECSN (whose effects are essentially identical to those for no kicks, as explained above) give an $e$-distribution which peaks at low eccentricities, while the large kicks characteristic of ICCSN give high eccentricities with the $e$-distribution peaking at a rather large value, as expected. We note that the positions of the peaks of the $e$-distributions obtained here for both ECSN and ICCSN are similar to those obtained for HMXBs (see Paper I). This is particularly true in the latter case, although the distribution for LMXBs appears to be much wider than that for HMXBs. We emphasize again here that the immediate post-SN 
$e$-distribution is not amenable to observation, though indirect clues on eccentricities of pre-LMXBs can be obtained from observations of individual X-ray binaries if they can be plausibly identified as pre-LMXBs, as described by Bhadkamkar \& Ghosh (2009).

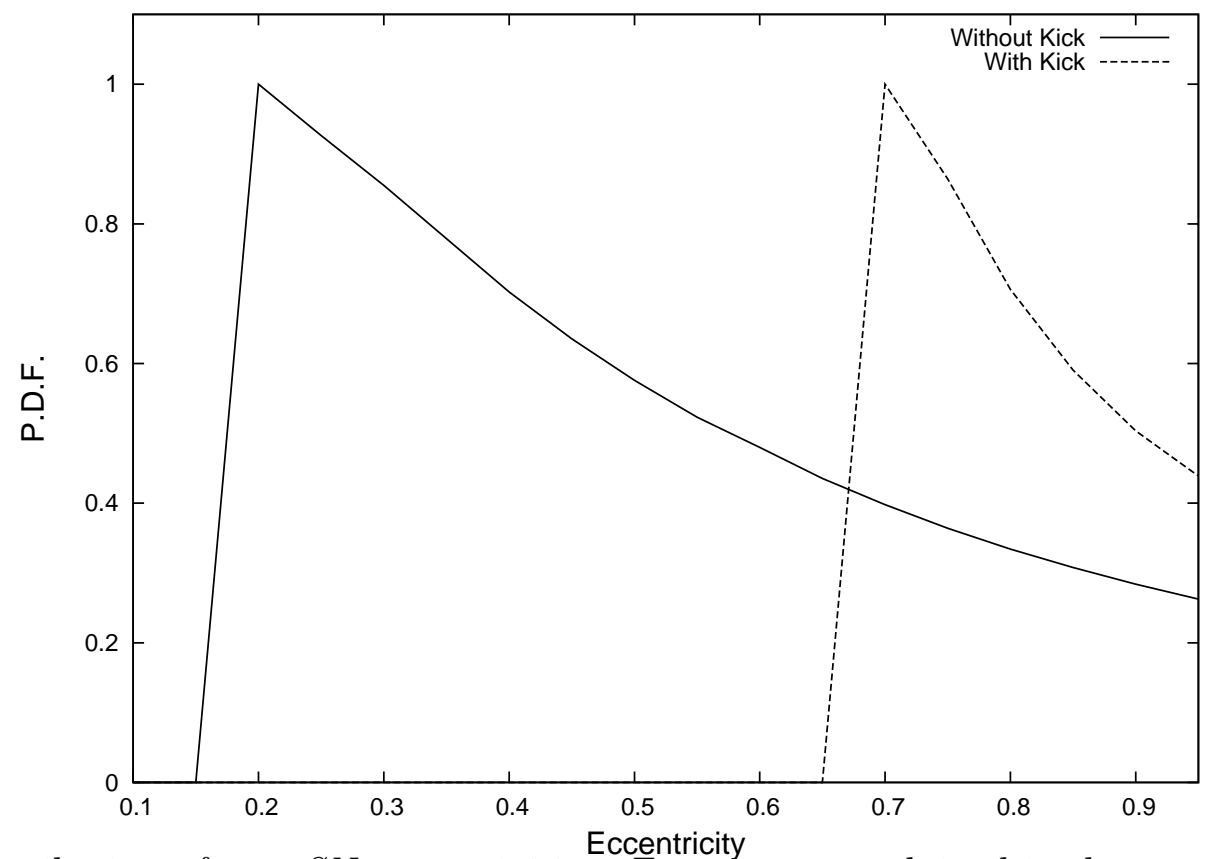

Fig. 3.-Distribution of post-SN eccentricities. For reasons explained in the text, the ECSN case is labeled "Without Kick", and the ICCSN case, "With Kick".

The change in the semimajor axis is obtained by solving Eq. (5) numerically for $\alpha$, which gives $a / a_{p s n}$. The Jacobian for this transformation is given by Eq.(7).

The primordial-binary PDF, $f_{\text {prim }}$ given by Eq.(8) can now be transformed into the pre-LMXB PDF using the Jacobian transformation, which can be schematically written as:

$$
f_{\text {ptid }}\left(M_{s}, a, e\right)=f_{\text {prim }}\left(M_{p}, q, a_{0}\right) J_{C E} J_{s n} J_{\text {tid }} .
$$

The pre-LMXB PDF is finally obtained by integrating $f_{p t i d}$ over eccentricity:

$$
f_{p l m}\left(M_{s}, a\right)=\int_{0}^{1} f_{p t i d} d e .
$$




\subsection{Properties of the pre-LMXB PDF}

The pre-LMXB PDF is a bivariate function of $M_{s}$ and $a$. We display this bivariate PDF in Figs, $4(a, b)$ and 5 ( $(a, b)$ for the four possible cases arising out of the two $q$-distributions (i.e., flat or steeply falling power-law) and the two SN-kick situations (i.e., no-kick/small ECSN kick or large ICCSN kick) that we explore in this work. In these 3-D displays, we have attempted to use the optimal viewing angles for bringing out the most essential features of the distributuion in each case, so that this viewing angle is not the same in all cases.
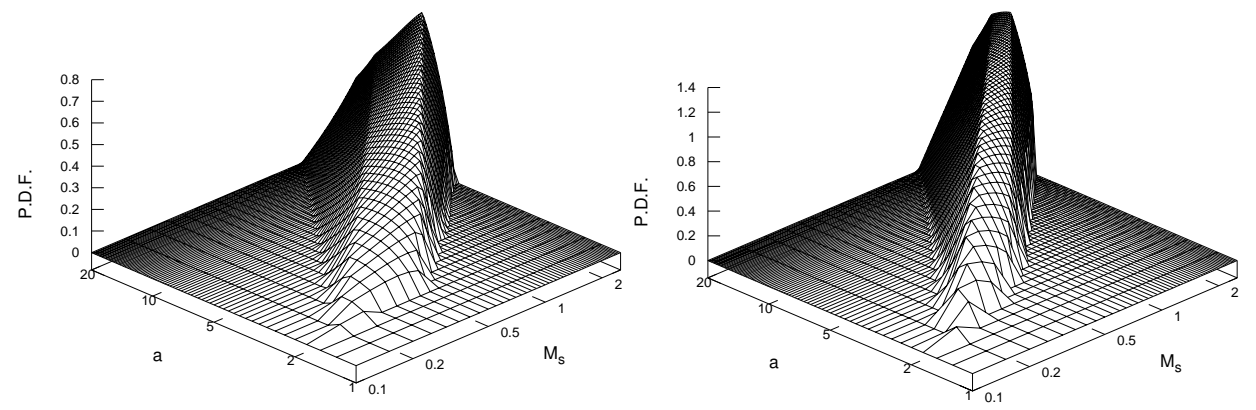

Fig. 4.-Bivariate pre-LMXB distribution as a function of $M_{s}$ and a for a flat q-distribution. Left (panel a): without kick, Right (panel b): with kick
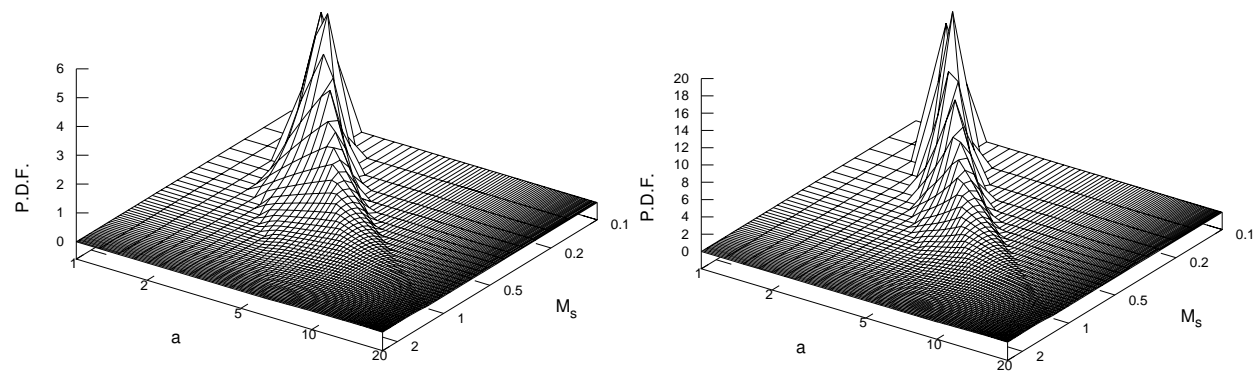

Fig. 5.- Bivariate pre-LMXB distribution as a function of $M_{s}$ and a for a falling power-law q-distribution. Left (panel a): without kick, Right (panel b): with kick

The individual (monovariate) PDF for a given variable can then be obtained by further integration of the above bivariate PDF over the other variable. Since $f_{\text {prim }}$ has been 
normalized over the allowed range for the viable pre-LMXB formation, $f_{p l m}$ is normalized automatically. However, any further integration requires the knowledge of the appropriate limits for the two parameters of the pre-LMXB systems. The range of $M_{s}$ is [0.1-2.5], which comes from various constraints described in Sec.4. The distribution of $a$ is obtained by integrating $f_{p l m}$ over this range of values of $M_{s}$. The integration range of $a$ is chosen as [0-20] for obtaining the PDF as a function of $M_{s}$. Note that the lower $a$-limit is not very restrictive, as the PDF must fall to zero at $a \approx 1.5 R_{c}$, so that Roche lobe contact does not occur just after the SN. The upper $a$-limit comes from the fact that wider systems will not come into Roche-lobe contact in a Hubble time. The actual value chosen is typical, corrsponding to the range of values of the pre-LMXB companion mass. We note here that $f_{p l m}$ does not fall to zero at this upper limit, so that we are excluding at this point a few systems which are not expected to reach the LMXB phase. Consequently, the individual (monovariate) PDF is not automatically normalised, unlike earlier.

The individual PDFs thus obtained depend upon the parameters which decide the strengths of the various steps involved in the formation of pre-LMXBs. These parameters are (a) the CE parameter (default value 1.0) (b) metallicity (default value 0.02) (c) exponent of the mass-ratio PDF, $\beta$, and (d) the scenario for the SN-kicks (i.e., no-kick/small ECSN kick or large ICCSN kick).

\subsubsection{Companion-mass distribution}

The distribution of the companion mass is not directly affected by the CE parameter and the metallicity. The effects of these parameters enter indirectly through the allowed phase-space area, since these parameters affect the allowed range of $a$. By contrast, the primordial mass-ratio distribution exponent $\beta$ affects the distribution of the companion mass directly. Fig. 6 shows the companion-mass PDF for $\beta=0$ (flat distribution) and $\beta=-2.7$ 


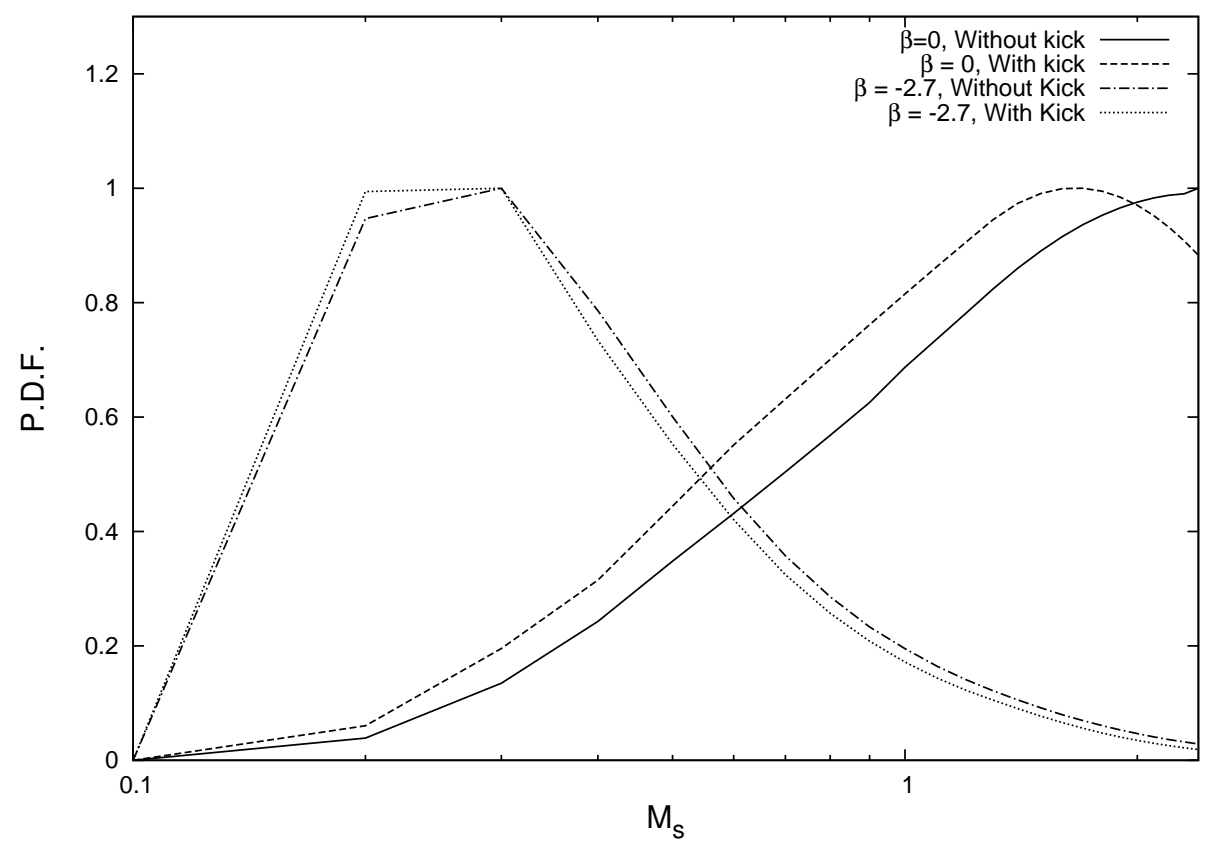

Fig. 6.- Distribution of the companion mass for the four cases displayed in Figs $4(a, b)$ and $5(a, b)$. Cases coded by line styles as indicated.

(power-law distribution), clearly demonstrating that a falling power-law distribution makes the $M_{s}$ distribution peak at much lower values of the companion mass and so makes the rise to this peak faster. On the other hand, the shape of the PDF changes little between the different kick-scenarios, particularly in the $\beta=-2.7$ case. The shapes of these PDFs are in agreement with those given in earlier works in the subject, i.e., Pfahl et al. (2003) for the $\beta=0$ case, and Kalogera \& Webbink (1998) for the $\beta=-2.7$ case. In the former case, the agreement is particularly striking. In the latter case, the authors gave their PDFs as bivariate 3D-plots, so that it is easier to compare our corresponding plots displayed in Figs $15(\mathrm{a}, \mathrm{b})$. The nearly-flat-top region in our $M_{s}$-distribution for $\beta=-2.7$ and large ICCSN kicks shows up as a "ridge" in our bivariate distribution of Fig (5), which can be compared with the corresponding feature in Fig.6 of Kalogera \& Webbink (1998).

Fig:7 shows the variation of the companion mass PDF for different values of the $\mathrm{CE}$ parameter. Drastic difference between $\alpha \lambda=0.5$ and 2 case can be seen. Whereas for 

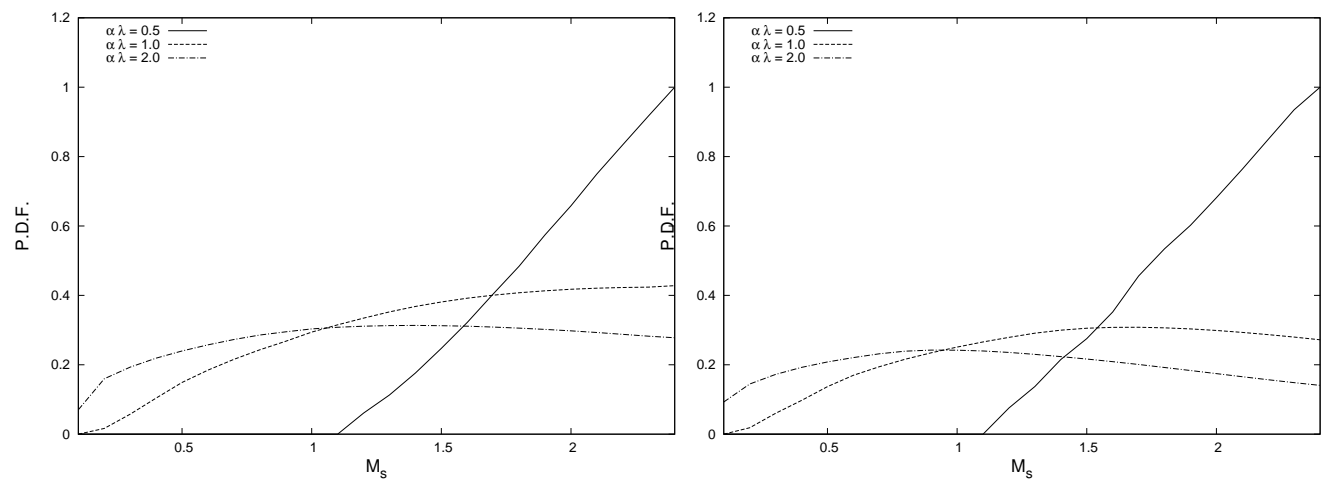

Fig. 7.- Distribution of the companion mass for different values of the CE parameter $\alpha \lambda$, whose values are coded by line styles as indicated. Left (Panel a): no-kick/small ECSN kick. Right (Panel b): large ICCSN kick.

$\alpha \lambda=0.5$ the PDF is linearly rising, for $\alpha \lambda=2.0$ it shows an early rising part which quickly saturates. The $\alpha \lambda=1$ case can be seen as an intermediate one. Again, we see that the inclusion of large kicks does not change the shape of the PDF drastically.
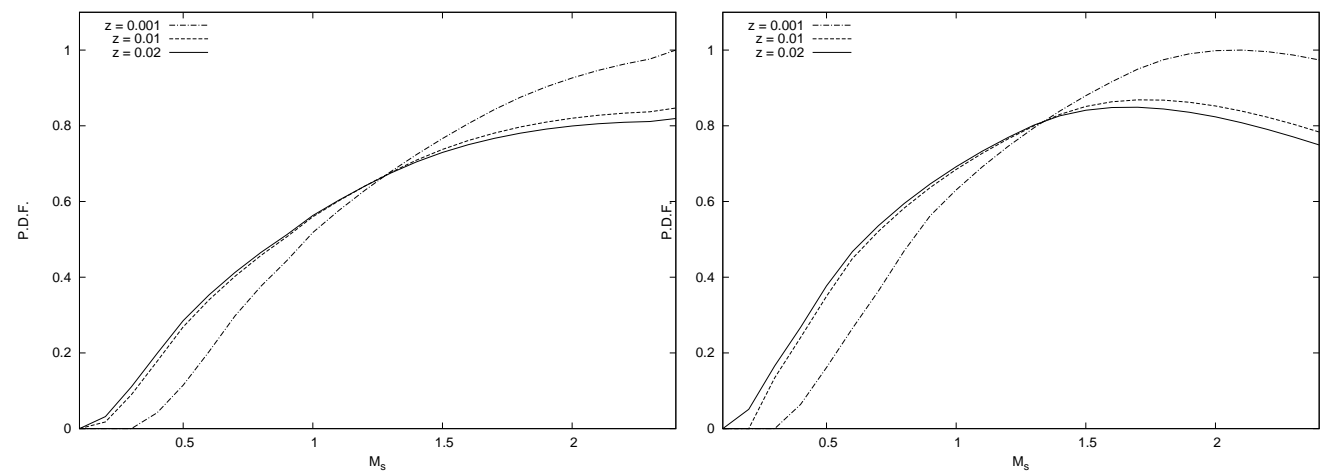

Fig. 8.- Distribution of the companion mass for different values of metallicity z, whose values are coded by line styles as indicated. Left (Panel a): no-kick/small ECSN kick. Right (Panel b): large ICCSN kick.

Variations in the metallicity affect the $M_{s}$-PDF more weakly than those in the CE-parameter. This is as expected, since the effects of both of these parameters are only through their influences on the allowed zones as explained above, and since the influences of the metallicity are much weaker. We further note that the $z=0.01$ and $z=0.02$ cases 
are not very different from each other.

\subsubsection{Distribution of orbital separation}

The distribution of the orbital separation $a$ is also affected by the above parameters. $\operatorname{Fig} 9(\mathrm{a}, \mathrm{b})$ shows the variation of the $a$-distribution with the CE parameter for the two SN-kick scenarios (note that this and the next two figures show the PDF plotted on a logarithmic $a$-scale, i.e., $P D F=d P / d \ln a$ is displayed as a function of $a$ plotted on a logarithmic scale). The peak of the distribution shifts to smaller $a$-values as the CE parameter increases, reflecting an increase in the allowed zone. The $a$-distribution is clearly not log-uniform for either kick-scenario.
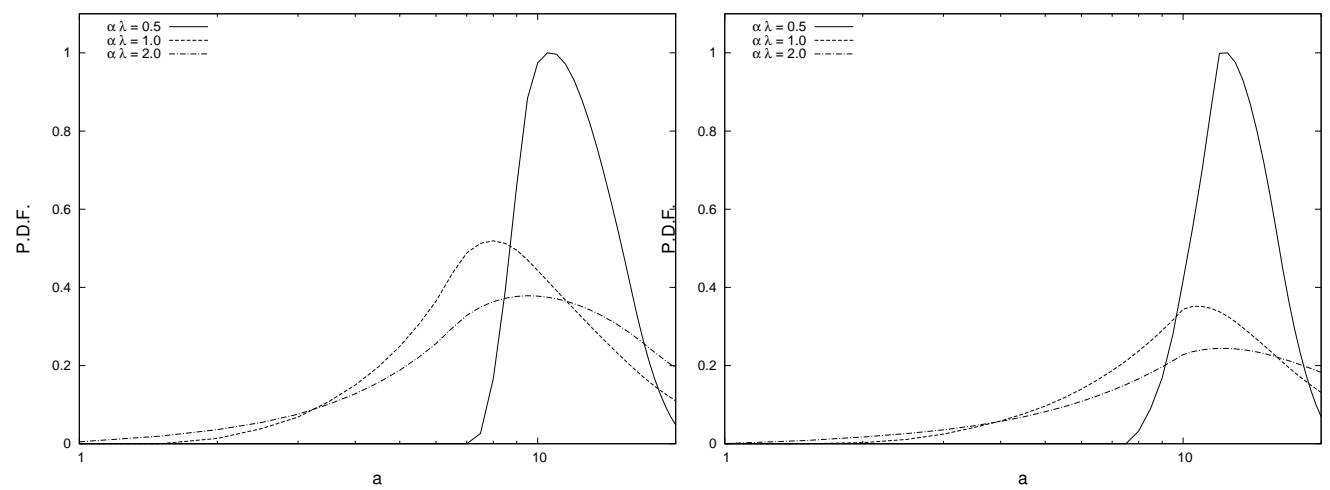

Fig. 9.- Distribution of the orbital separation a for different values of the CE parameter, these values being coded by line styles as indicated. Left (Panel a): no-kick/small ECSN kick. Right (Panel b): large ICCSN kick.

Fig.10(a, b) shows the variation of $a$-distribution with metallicity. Little change is seen for different values of the metallicity, with the peak shifting slightly to the left at lower metallicities. Again, we see little change in the shape of the PDF after the inclusion of SN-kicks.

Fig. 11 shows the effect of varying $\beta$, i.e., the exponent of the $q$-distribution, on the 

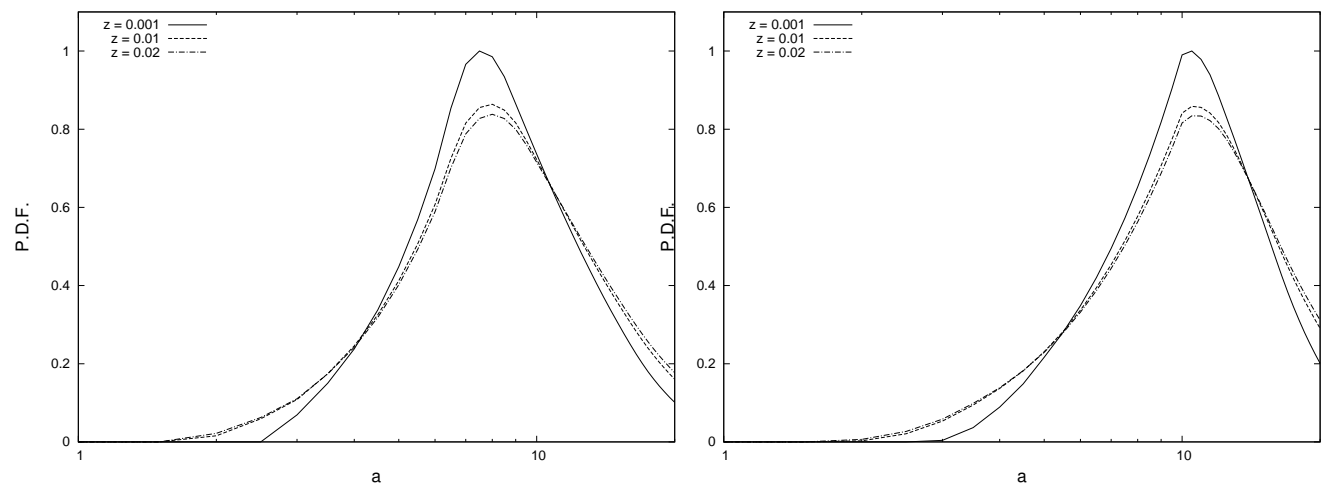

Fig. 10.- Distribution of the orbital separation for different values of metallicity, these values being coded by line styles as indicated. Left (Panel a): no-kick/small ECSN kick. Right (Panel b): large ICCSN kick.

$a$-distribution, which is quite strong. The steeply-falling power law with $\beta=-2.7$ shifts the distribution peak to much smaller values of a compared to the situation for the flat $(\beta=0) q$-distribution. Again, we find here close similarities to previous results in the literature, i.e., Pfahl et al. (2003) for the $\beta=0$ case, and Kalogera \& Webbink (1998) for the $\beta=-2.7$ case, and again the former similarity is particularly striking. We emphasize that the general rise-and-fall shape of the $a$-distribution for pre-LMXBs seems both quite generic and confirmed by all previous calculations known to us, and that this shape stands in contrast to the generically flat or nearly-flat shape at intermediate $a$ 's that we found for pre-HMXBs and HMXBs in Paper I.

\section{Formation Rate}

In this section, we describe our recipe for calculating the formation rate of pre-LMXBs, which serves as an input to the calculation of the LMXB X-ray luminosity function (XLF) described in Paper III. The first point we notice is that the various steps in the formation process of pre-LMXBs described in previous sections do not take equal amounts of time. 


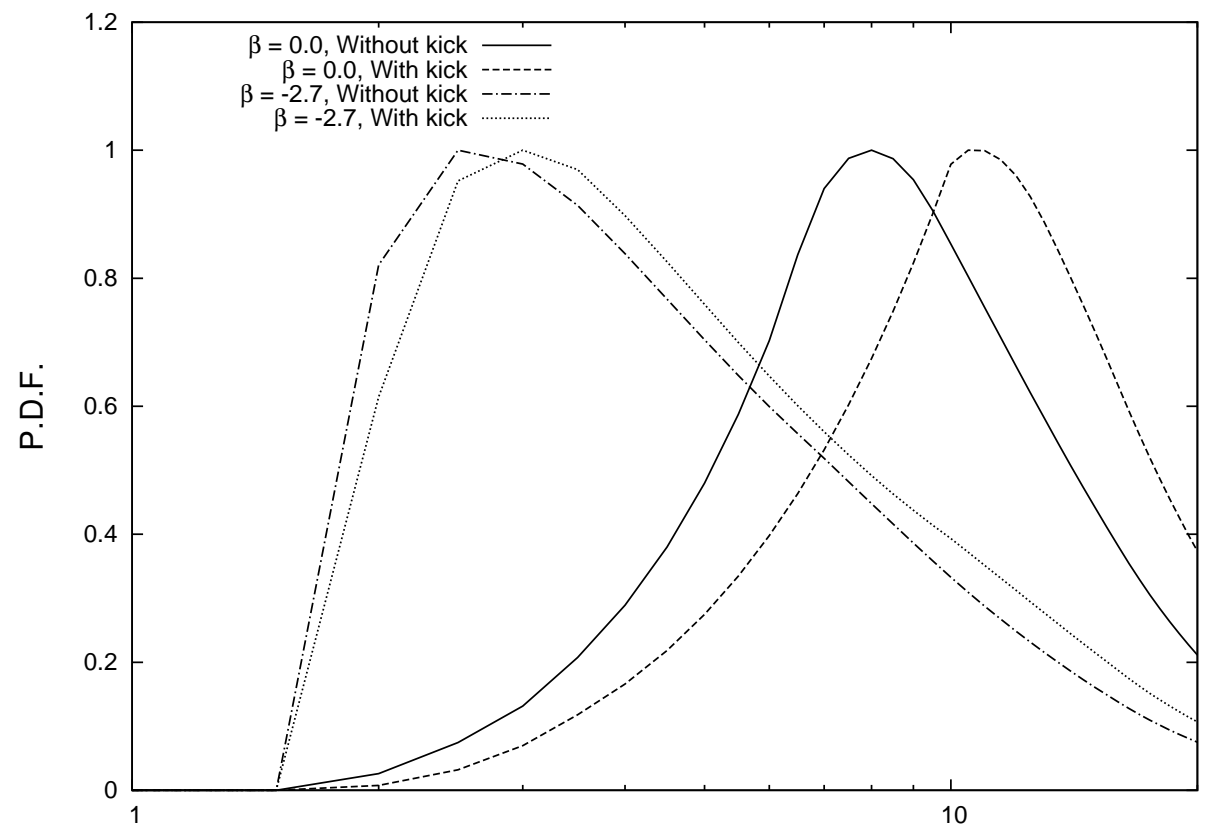

Fig. 11. - Distribution of the orbital separation a for four cases corresponding to two different values of $\beta$ and two different SN-kick scenarios. Each case coded by line style, as indicated.

The three timescales that are of relevance here are (a) $\tau_{\text {prim }}$, the timescale for the primordial binary to reach the CE phase, (b) $\tau_{\text {postCE}}$, the timescale of evolution of the post-CE binary upto the SN explosion, and, (c) $\tau_{\text {tid }}$, the timescale of tidal evolution of the post-SN binary. We first note that $\tau_{p o s t C E}$, which essentially is the timescale of evolution of a He-star, is much shorter than the other two timescales. $\tau_{\text {tid }}$ can have a considerable range, depending upon the initial eccentricity of the system just after the SN. Typical tidal timescales given in literature are $\sim 10^{5}$ years (Zahn 1977; Bhadkamkar \& Ghosh 2009). We assume here that the typical range given by these authors works well for the entire relevant range. In that case, $\tau_{\text {tid }}$ may be an order of magnitude smaller than $\tau_{\text {prim }}$, which is the timescale of the evolution of the primary, $\sim 10^{6-7}$ years. Thus the dominant timescale in the evolution of a pre-LMXB is $\tau_{\text {prim }}$.

The formation rate of pre-LMXBs is related to the star formation rate (SFR). Since the SFR evolves on timescales that are much longer than $\tau_{\text {prim }}$ (typically $\sim 10^{9}$ years), we can 
treat the SFR to be quasistatic during pre-LMXB formation, and say that the formation rate of pre-LMXBs is roughly equal to the SFR at a (small) timelag of $\tau_{\text {prim }}$. Since we are ultimately interested in the evolution of these systems to the LMXB phase, we need to take into account the formation rates over a long span of time when computing the evolution of pre-LMXBs into LMXBs. Typical evolutionary timescale of LMXB systems is $\sim 10^{9}$ years, so that we need to consider the evolutionary history of the SFR over timescales of Gyrs. Evolution of the SFR has been studied in great detail in the literature over the last fifteen years or so, using multiwavelength studies of galaxies as well as theoretical investigations of the underlying evolutionary processes (Madau et al. 1996, 1998; Pettini et al. 1998; Blain et al. 1999; Hartwick 2004). The SFR profiles fitted to the data can be generally divided into two classes: peak-type and anvil-type. (See Blain et al. (1999) for a detailed summary of the models in each class). These models generally provide the SFR as a function of the redshift $z$, which needs to be converted into the lookback time for our purposes here. The relation $z \equiv z(t)$ is dependent on the details of the cosmology assumed: we do not go into the details of cosmological models here, but rather assume the standard modern prescription in the literature.

We present here a general method of calculating the formation rate of pre-LMXBs, applicable to any form of SFR profile. We note first that $\tau_{\text {prim }}$ is roughly equal to the main sequence life time of the primary, which is a function of the mass of the primary and can be approximated by $\tau_{\text {prim }} \approx 12 M_{p}^{-2.5}$ Gyr for the primary mass range of interest in this work (See appendix C of Ghosh (2007)). $M_{p}$ can be calculated using the inverse transformations, but we need all three parameters describing the final stage to calculate $M_{p}$. The net formation rate of the pre-LMXBs at any given time is given by $S F R\left(t-\tau_{\text {prim }}\right)$. The formation rate for specific values of $M_{s}$ and $a$ can be calculated by multiplying the formation rate with the PDF and integrating this product over all eccentricities, since the net formation rate is dependent on eccentricity through $\tau_{\text {prim }}$. Thus the formation rate as a 
function of $M_{s}$ and $a$ can be written as

$$
R\left(M_{s}, a ; t\right)=\int_{0}^{1} \operatorname{SFR}\left(\mathrm{t}-\tau_{\text {prim }}\right) \mathrm{f}_{\mathrm{tid}}\left(\mathrm{M}_{\mathrm{s}}, \mathrm{a}, \mathrm{e}\right) \mathrm{de} .
$$

The formation rate given by Eq.(11) can be used as a starting point for studying the evolution of LMXBs, which we do in Paper III. As noted above, one needs to know $R\left(M_{s}, a ; t\right)$ for at least a few Gyr back from present epoch if one wants to calculate the properties of the current population of LMXBs. The importance of cosmic star formation history (SFH) on collective properties of LMXBs has been studied for more than a decade now (White \& Ghosh 1998; Ghosh \& White 2001). The work of these authors was a first step in this direction, which described the evolution of the total number of these systems without considering the distributions of the system parameters. Our scheme of calculations presented here and in Paper III of this series, where we follow the evolution of LMXBs, enables us to study various collective properties of LMXB populations as distributions of luminosity (i.e., XLF) and orbital period.

\section{Discussion}

We have described in this paper a method for calculating the formation rate of pre-LMXBs from given distributions of primordial binaries and given star formation rates. We studied the pre-LMXB PDF as a function of the companion mass and the orbital separation for various values of CE parameter, SN-kick scenario, and other parameters. The main conclusions of this work can be summarised as follows:

1. LMXB formation is a very tightly constrained process. We showed that the constraints suggested by Kalogera \& Webbink can be transformed into constraints on primordial binaries and so demonstrated how only a small allowed region in the phase space 
of primordial binaries is able to produce pre-LMXBs and then possibly LMXBs (if further conditions are satisfied, e.g., attainment of Roche lobe contact within a Hubble time). We showed that the CE parameter was a major factor affecting the allowed phase space, so that a good understanding of the CE process was essential for modeling the collective properties of LMXBs.

2. The PDF of pre-LMXBs was studied in a bivariate form,i.e., as a function of $M_{c}$ and $a$ as well as in monovariate forms for each of these variables. These PDFs were shown to agree with the results of earlier studies in this field. It was shown that a power-law distribution of the primordial mass ratio $q$ can lead to very different PDFs for different values of the exponent $\beta . \beta=0$ leads to a larger number of wider systems, and a companion mass distribution skewed towards the higher end. This would naturally lead to a larger number of LMXB systems harboring giant companions. On the contrary, $\beta=-2.7$ leads to a larger number of compact systems with smaller-mass companions. This would lead to a larger number of LMXB systems with main-sequence companions. We take up these questions in more detail in Paper III.

3. The effects of the metallicity of the primordial primary and of the inclusion of natal SN-kicks were also studied. The former effects are generally small. For the latter, significant effects come only for ICCSN-kicks, as the ECSN-kicks are so small as to give results essentially identical to those for the no-kick scenario.

In the next paper (Paper III of the series), we proceed from the pre-LMXB formation rate found in this paper to a computation of the expected LMXB XLF, which we then compare with the observed LMXB XLF with a view to understanding and constraining the essential processes of pre-LMXB and LMXB formation and evolution. We re-emphasize that this effort should be regarded as a proof-of-principle type of exercise to understand the 
basic physics underlying the LMXB XLF, similar to what we did in Paper I for the HMXB XLF. 


\section{REFERENCES}

Bhadkamkar, H., \& Ghosh, P. 2009, A\&A, 506, 1297

-. 2012, ApJ, 746, 22

Blain, A. W., Smail, I., Ivison, R. J., \& Kneib, J.-P. 1999, MNRAS, 302, 632

De Marco, O., Passy, J.-C., Moe, M., Herwig, F., Mac Low, M.-M., \& Paxton, B. 2011, MNRAS, 411, 2277

Dewi, J. D. M., \& Tauris, T. M. 2000, A\&A, 360, 1043

Eggleton, P. P. 1983, ApJ, 268, 368

Ghosh, P. 2007, Rotation and Accretion Powered Pulsars (World Scientific Publishing)

Ghosh, P., \& White, N. E. 2001, ApJ, 559, L97

Gilfanov, M. 2004, MNRAS, 349, 146

Gilfanov, M., Grimm, H.-J., \& Sunyaev, R. 2004a, Nuclear Physics B Proceedings Supplements, 132, 369

-. 2004b, MNRAS, 347, L57

Grimm, H.-J., Gilfanov, M., \& Sunyaev, R. 2002, A\&A, 391, 923

-. 2003, MNRAS, 339, 793

Hartwick, F. D. A. 2004, ApJ, 603, 108

Hurley, J. R., Pols, O. R., \& Tout, C. A. 2000, MNRAS, 315, 543

Hut, P. 1981, A\&A, 99, 126 
Ivanova, N. 2011, ApJ, 730, 76

Kalogera, V. 1996, ApJ, 471, 352

—. 1998, ApJ, 493, 368

Kalogera, V., \& Webbink, R. F. 1998, ApJ, 493, 351

Kim, D.-W., \& Fabbiano, G. 2004, ApJ, 611, 846

-. 2010, ApJ, 721, 1523

Kroupa, P., Tout, C. A., \& Gilmore, G. 1993, MNRAS, 262, 545

Kroupa, P., \& Weidner, C. 2003, ApJ, 598, 1076

Liu, Q. Z., van Paradijs, J., \& van den Heuvel, E. P. J. 2007, A\&A, 469, 807

Madau, P., Ferguson, H. C., Dickinson, M. E., Giavalisco, M., Steidel, C. C., \& Fruchter, A. 1996, MNRAS, 283, 1388

Madau, P., Pozzetti, L., \& Dickinson, M. 1998, ApJ, 498, 106

Öpik, E. 1924, Publications of the Tartu Astrofizica Observatory, 25, 1

Pettini, M., Kellogg, M., Steidel, C. C., Dickinson, M., Adelberger, K. L., \& Giavalisco, M. 1998, ApJ, 508, 539

Pfahl, E., Rappaport, S., \& Podsiadlowski, P. 2003, ApJ, 597, 1036

Podsiadlowski, P., Rappaport, S., \& Pfahl, E. D. 2002, ApJ, 565, 1107

Pylyser, E., \& Savonije, G. J. 1988, A\&A, 191, 57

Pylyser, E. H. P., \& Savonije, G. J. 1989, A\&A, 208, 52 
Rucinski, S. M. 1988, AJ, 95, 1895

Salpeter, E. E. 1955, ApJ, 121, 161

Sana, H., \& Evans, C. J. 2010, arXiv:astro-ph/1009.4197

Taam, R. E., \& Sandquist, E. L. 2000, ARA\&A, 38, 113

van den Heuvel, E. P. J. 1983, in Accretion-Driven Stellar X-ray Sources, ed. W. H. G. Lewin \& E. P. J. van den Heuvel, 303

van den Heuvel, E. P. J. 1991, in NATO ASIC Proc. 344: Neutron Stars, ed. J. Ventura \& D. Pines, $171-+$

van den Heuvel, E. P. J. 1992, in X-Ray Binaries and the Formation of Binary and Millisecond Radio Pulsars, 233-256

van den Heuvel, E. P. J. 2001, in The Neutron Star - Black Hole Connection, ed. C. Kouveliotou, J. Ventura, \& E. van den Heuvel, 173

Webbink, R. F. 1984, ApJ, 277, 355

Webbink, R. F. 2008, in Astrophysics and Space Science Library, Vol. 352, Astrophysics and Space Science Library, ed. E. F. Milone, D. A. Leahy, \& D. W. Hobill, 233-+

White, N. E., \& Ghosh, P. 1998, ApJ, 504, L31

Willems, B., \& Kolb, U. 2004, A\&A, 419, 1057

Zahn, J.-P. 1977, A\&A, 57, 383 